Convenient test for the weak metric regularity of a non strictly Fréchet differentiable mappings

\author{
S. Lahrech
}




\title{
CONVENIENT TEST FOR THE WEAK METRIC REGULARITY OF A NON STRICTLY FRÉCHET DIFFERENTIABLE MAPPINGS
}

\author{
S. LAHRECH
}

Received 26 September, 2005

\begin{abstract}
Using a recent extension of Fréchet differentiability (approach of Taylor mappings, see [1]), the notion of Clarke subdifferential in binormed spaces, and the notion of closed pair of multifunctions, we present a convenient test for the weak metric regularity of a non-strictly Fréchet differentiable functions in terms of the surjectivity of its Taylor strict derivative. As an application, we give an example of a non-strictly Fréchet differentiable function for which the given test works.
\end{abstract}

2000 Mathematics Subject Classification: 49J52, 49L25, 49J40, 49J50

Keywords: weak metric regularity, Clarke subdifferential in binormed spaces, strictly Taylor differentiable mappings, strictly Fréchet differentiable mappings, closed pair of multifunctions

\section{AN INTRODUCTION TO METRIC REGULARITY}

Throughout this section, we consider an open set $U$ in a normed space $\left(E,\|\cdot\|_{1}\right)$, a closed set $S \subset U$, a Euclidean space $(Y,\|\cdot\|)$, and a continuous map $h: U \rightarrow Y$.

Let $x \in S, T_{S}^{1}(x)$ the Clarke tangent cone to $S$ at $x$ with respect to the norm $\|\cdot\|_{1}$, $d_{S}^{1}$ the distance function to the set $S$ with respect to the norm $\|\cdot\|_{1}$.

The calculation of $K_{h^{-1}(h(x))}^{1}$ contingent cone to $h^{-1}(h(x))$ at $x$ with respect to the norm $\|\cdot\|_{1}$ requires first to bound the distance of a point $z$ to the set $h^{-1}(h(x))$ in terms of the function value $h(z)$. This leads us to the notion of metric regularity.

We say $h$ is $\|\cdot\|_{1}$ weakly metrically regular on $S$ at the point $x$ if there is a real constant $K$ such that

$$
d_{S \cap h^{-1}(h(x))}^{1}(z) \leq K\|h(z)-h(x)\|
$$

for all $z$ close to $x$ with respect to the norm $\|\cdot\|_{1}$. If $h$ is $\|\cdot\|_{1}$ strictly Fréchet differentiable at $x$, we can present a convenient condition for weak metric regularity as follows:

Theorem 1. If $h$ is $\|\cdot\|_{1}$ strictly Fréchet differentiable at the point $x$ in $S$ and $\nabla h(x)\left(T_{S}^{1}(x)\right)=Y$ then $h$ is $\|\cdot\|_{1}$ weakly metrically regular on $S$ at $x$.

A pretty application of the above theorem is the classical Liusternik theorem. 
Theorem 2. If $h$ is $\|\cdot\|_{1}$ strictly Fréchet differentiable at the point $x$ and $\nabla h(x)$ is surjective then the set $h^{-1}(h(x))$ is tangentially regular at $x$ and $K_{h^{-1}(h(x))}^{1}=$ $N(\nabla h(x))$, where $N(\cdot)$ denote the null space.

Using a recent extension of Fréchet differentiability (approach of Taylor mappings, see [1]) and the notion of Clarke subdifferential in binormed spaces, we want to establish the same conclusion of the theorem 1 for non $\|\cdot\|_{1}$ strictly Fréchet differentiable mappings.

For this, we pause to recall some terminology before proving the main result.

\section{STRICTLY TAYLOR DifFERENTIABLE MAPPINGS}

Let $\|\cdot\|_{1},\|\cdot\|_{2}$ two norms defined on a linear space $E$ such that $\left(E,\|\cdot\|_{2}\right)$ is a Banach space and, for some $c>0$, the condition $\|\cdot\|_{1} \leq c\|\cdot\|_{2}$ holds.

Let $U$ an open subset of $\left(E,\|\cdot\|_{1}\right), S$ a closed subset of $\left(E,\|\cdot\|_{1}\right)$ such that $S \subset U$, $(Y,\|\cdot\|)$ is a Euclidean space, $h: U \rightarrow Y$.

According to [1], we say that $h$ is a $\left(\|\cdot\|_{1},\|\cdot\|_{2}\right)$ strictly Taylor differentiable mapping at a point $x \in U$ if there exists a continuous linear operator from $\left(E,\|\cdot\|_{2}\right)$ to $(Y,\|\cdot\|)$ such that

$$
h\left(x^{\prime}+s\right)-h\left(x^{\prime}\right)-L s=o\left(\|s\|_{2}\right)
$$

in $(Y,\|\cdot\|)$ as $x^{\prime} \rightarrow_{\|\cdot\|_{1}} x, s \rightarrow_{\|\cdot\|_{1}} 0$. Let us first note that such $L$ is unique since $h$ is $\|\cdot\|_{2}$ Fréchet differentiable at $x$. If $h$ is $\left(\|\cdot\|_{1},\|\cdot\|_{2}\right)$ strictly differentiable at $x \in U$, then we set $\nabla h(x)=L$.

Even if $h$ is a $\left(\|\cdot\|_{1},\|\cdot\|_{2}\right)$ strictly Taylor differentiable mapping at a point $x, h$ is not necessarily $\|\cdot\|_{1}$ Fréchet differentiable at $x$. But if $\|\cdot\|_{1}$ is equivalent to the norm $\|\cdot\|_{2}$, then $h$ is necessarily strictly $\|\cdot\|_{1}$ Fréchet differentiable at $x$.

So the notion of strict differentiability in terms of Taylor strengthens and generalizes the elegant notion of strict Fréchet differentiability.

In order to generalize the classical Theorem 1, we extend the notion of Clarke subdifferentiability in a normed spaces to binormed spaces.

\section{Clarke SUbDifFERENTIAL IN BINORMED SPACES}

Throughout this section we consider a binormed space $\left(E,\|\cdot\|_{1},\|\cdot\|_{2}\right)$ such that $\left(E,\|\cdot\|_{2}\right)$ is a Banach space and, for some $c>0$, the inequality $\|\cdot\|_{1} \leq c\|\cdot\|_{2}$ holds, an open set $U$ of a normed space $\left(E,\|\cdot\|_{1}\right)$, a closed subset $S$ of $\left(E,\|\cdot\|_{1}\right)$ such that $S \subset U$, a Euclidean space $(Y,\|\cdot\|)$, a locally Lipschitzian around $\bar{x} \in U$ with respect to the norm $\|\cdot\|_{2}$ mapping $h: U \rightarrow(Y,\|\cdot\|)$, and a $\|\cdot\|_{2}$ locally Lipschitzian real function $h_{2}$ around $\bar{x} \in U$.

Let $v$ any vector in $E$. The Clarke generalized directional derivative of $h_{2}$ at $\bar{x}$ in the direction $v$ with respect to the norm $\|\cdot\|_{2}$, denoted $h_{2}^{0,2}(\bar{x}, v)$, is defined as 
follows:

$$
h_{2}^{0,2}(\bar{x}, v)=\limsup _{x^{\prime} \rightarrow \|_{\|} \bar{x}, t \downarrow 0} \frac{h_{2}\left(x^{\prime}+t v\right)-h_{2}\left(x^{\prime}\right)}{t} .
$$

The Clarke subdifferential of $h_{2}$ at $\bar{x}$ with respect to the norm $\|\cdot\|_{2}$, denoted $\partial_{0}^{2} h_{2}(\bar{x})$, is the subset of $\left(E,\|\cdot\|_{2}\right)^{\prime}$ given by

$$
\partial_{0}^{2} h_{2}(\bar{x})=\left\{\xi \in\left(E,\|\cdot\|_{2}\right)^{\prime}: h_{2}^{0,2}(\bar{x}, v) \geq\langle\xi, v\rangle \forall v \in E\right\} .
$$

Analogously, the Clarke subdifferential of $h_{2}$ at $\bar{x}$ with respect to the pair of norms $\left(\|\cdot\|_{1},\|\cdot\|_{2}\right)$, denoted $\partial_{0}^{1,2} h_{2}(\bar{x})$, is the subset of $\left(E,\|\cdot\|_{2}\right)^{\prime}$ given by

$$
\partial_{0}^{1,2} h_{2}(\bar{x})=\left\{\xi \in\left(E,\|\cdot\|_{2}\right)^{\prime}: h_{2}^{0,1}(\bar{x}, v) \geq\langle\xi, v\rangle \forall v \in E\right\} .
$$

Notice that the Clarke subdifferentials $\partial_{0}^{2} h_{2}(\bar{x})$ and $\partial_{0}^{1} h_{2}(\bar{x})$ are smaller than the generalized Clarke subdifferential $\partial_{0}^{1,2} h_{2}(\bar{x})$. For a point $x$ in $S$, the generalized Clarke tangent cone to $S$ at $x$ with respect to the norms $\left(\|\cdot\|_{1},\|\cdot\|_{2}\right)$, denoted $T_{S}^{1,2}(x)$, is the subset of $E$ given by

$$
T_{S}^{1,2}(x)=\left(\operatorname{cl}\left(R^{+} \partial_{0}^{1,2} d_{S}^{2}(x)\right)^{-},\right.
$$

where cl denotes the $* \sigma\left(\left(E,\|\cdot\|_{2}\right)^{\prime}, E\right)$ closure.

Analogously, the generalized Clarke normal cone to $S$ at $x$ with respect to the norms $\left(\|\cdot\|_{1},\|\cdot\|_{2}\right)$, denoted $N_{S}^{1,2}(x)$, is the subset of $\left(E,\|\cdot\|_{2}\right)^{\prime}$ given by

$$
N_{S}^{1,2}(x)=\operatorname{cl}\left(R^{+} \partial_{0}^{1,2} d_{S}^{2}(x)\right),
$$

where cl denotes $* \sigma\left(\left(E,\|\cdot\|_{2}\right)^{\prime}, E\right)$ closure.

From the above definitions, we deduce immediately that the generalized tangent cone $T_{S}^{1,2}(x)$ is smaller than the tangent cones $T_{S}^{2}(x)$ and $T_{S}^{1}(x)$.

The first assertion below reiterates that if $h_{2}$ is a $\|\cdot\|_{2}$ locally Lipschitzian real function on $U$, then the pair of multifunctions $\left(\partial_{0}^{2} h_{2}, \partial_{0}^{1,2} h_{2}\right)$ is closed from $\left(U,\|\cdot\|_{1}\right)$ to $\left(\left(E,\|\cdot\|_{2}\right)^{\prime}, * \sigma\left(\left(E,\|\cdot\|_{2}\right)^{\prime}, E\right)\right)$ in the following sense: if $x_{i}$ and $\xi_{i}$ are sequences in $U$ and $\left(E,\|\cdot\|_{2}\right)^{\prime}$ such that $\xi_{i} \in \partial_{0}^{2} h_{2}\left(x_{i}\right), x_{i}$ converges to $x$ with respect to the norm $\|\cdot\|_{1}$, and $\xi_{i}$ converges to $\xi$ for the weak topology $* \sigma\left(\left(E,\|\cdot\|_{2}\right)^{\prime}, E\right)$, then $\xi \in$ $\partial_{0}^{1,2} h_{2}(x)$.

Proposition 1. Assume that $h_{2}$ is a $\|\cdot\|_{2}$ locally Lipschitzian real function on $U$. Then the pair of multifunctions $\left(\partial_{0}^{2} h_{2}, \partial_{0}^{1,2} h_{2}\right)$ is closed from $\left(U,\|\cdot\|_{1}\right)$ to $\left(\left(E,\|\cdot\|_{2}\right)^{\prime}\right.$, $\left.* \sigma\left(\left(E,\|\cdot\|_{2}\right)^{\prime}, E\right)\right)$.

Proof. Let $x_{i}$ and $\xi_{i}$ be sequences in $U$ and $\left(E,\|\cdot\|_{2}\right)^{\prime}$ such that $\xi_{i} \in \partial_{0}^{2} h_{2}\left(x_{i}\right)$, $x_{i}$ converges to $x$ with respect to the norm $\|\cdot\|_{1}$, and $\xi_{i}$ converges to $\xi$ for the weak topology $* \sigma\left(\left(E,\|\cdot\|_{2}\right)^{\prime}, E\right)$.

Let any $v \in E$ be given. Then $\left\langle\xi_{i}, v\right\rangle$ converges to $\langle\xi, v\rangle$. One has $h_{2}^{0,2}\left(x_{i}, v\right) \geq$ $\left\langle\xi_{i}, v\right\rangle$, which implies that $h_{2}^{0,1}\left(x_{i}, v\right) \geq\left\langle\xi_{i}, v\right\rangle$. 
By the upper semicontinuity of $h_{2}^{0,1}$ with respect to the norm $\|\cdot\|_{1}$, we deduce that $h_{2}^{0,1}(x, v) \geq\langle\xi, v\rangle$. Since $v$ is arbitrary, $\xi$ belongs to $\partial_{0}^{1,2} h_{2}(x)$.

Now we can give the main result.

\section{A CONVENIENT TEST FOR WEAK METRIC REgUlaRity OF $\left(\|\cdot\|_{1},\|\cdot\|_{2}\right)$ STRICTLY TAYLOR DIFFERENTIABLE MAPPINGS}

Assume that all the hypothesis of Section 3 are satisfied and suppose also that $\left(E,\|\cdot\|_{2}\right)$ is a separable Banach space. We can now present a convenient condition for weak metric regularity of $\left(\|\cdot\|_{1},\|\cdot\|_{2}\right)$ strictly Taylor differentiable functions.

Theorem 3 (Surjectivity and metric regularity). If $h$ is $\|\cdot\|_{2}$ strictly differentiable at the point $x$ in $S$ and $\nabla h(x)\left(T_{S}^{1,2}(x)\right)=Y$ then $h$ is $\|\cdot\|_{1}$ weakly metrically regular on $S$ at $x$.

Let us remark that in our theorem, to give a convenient test for the $\|\cdot\|_{1}$ weak metric regularity of a function at a point in terms of the surjectivity of its $\|\cdot\|_{2}$ strict derivative there, we do not require that $h$ is $\|\cdot\|_{1}$ strictly differentiable at the point $x$ as in the classical Theorem 1 .

Proof. Without loss of generality, suppose that $x=0$ and $h(0)=0$. If $h$ is not $\|\cdot\|_{1}$ weakly metrically regular on $S$ at the point 0 then there is a sequence $v_{r} \rightarrow 0$ in $\left(S,\|\cdot\|_{1}\right)$ such that $h\left(v_{r}\right) \neq 0$ for all $r$, and a real sequence $\delta_{r} \downarrow 0$ such that the function $\|h(\cdot)\|+\delta_{r}\left\|\cdot-v_{r}\right\|_{1}$ is minimized on $S$ at $v_{r}$. Denoting the local Lipschitz constant by $L$, we deduce from the nonsmooth calculus and the Exact penalization theorem the condition

$$
0 \in \partial_{0}^{2}(\|h\|)\left(v_{r}\right)+\delta_{r} B^{*}+L \partial_{0}^{2} d_{S}^{2}\left(v_{r}\right),
$$

Where $B^{*}$ denote the unit ball in $\left(E,\|\cdot\|_{2}\right)^{\prime}$.

Hence there are elements $u_{r}$ of $\partial_{0}^{2}(\|h\|)\left(v_{r}\right)$ and $w_{r}$ of $L \partial_{0}^{2} d_{S}^{2}\left(v_{r}\right)$ such that $u_{r}+$ $w_{r}$ approaches zero with respect to the dual norm $\|\cdot\|_{2}^{\prime}$.

By choosing a subsequence we can assume $\left\|h\left(v_{r}\right)\right\|^{-1} h\left(v_{r}\right) \rightarrow y \neq 0$. Consequently, $u_{r} \rightarrow(\nabla h(0))^{*} y$ for the weak topology $* \sigma\left(\left(E,\|\cdot\|_{2}\right)^{\prime}, E\right)$. Since the pair of multifunctions $\left(\partial_{0}^{2} d_{S}^{2}, \partial_{0}^{1,2} d_{S}^{2}\right)$ is closed at 0 , we deduce $-(\nabla h(0))^{*} y \in L \partial_{0}^{1,2} d_{S}^{2}(0) \subset$ $N_{S}^{1,2}(0)$. However, by assumption there is a nonzero element $p$ of $T_{S}^{1,2}(0)$ such that $\nabla h(0) p=-y$, so we arrive at the contradiction

$$
0 \geq\left\langle p,-(\nabla h(0))^{*} y\right\rangle=\langle\nabla h(0) p,-y\rangle=\|y\|>0,
$$

which completes the proof.

Corollary 1. If $h$ is $\left(\|\cdot\|_{1},\|\cdot\|_{2}\right)$ strictly differentiable at the point $x$ in $S$ and $\nabla h(x)\left(T_{S}^{1,2}(x)\right)=Y$ then $h$ is $\|\cdot\|_{1}$ weakly metrically regular on $S$ at $x$. 
Proof. Since $h$ is $\left(\|\cdot\|_{1},\|\cdot\|_{2}\right)$ strictly differentiable at the point $x$ then $h$ is $\|\cdot\|_{2}$ strictly differentiable at the point $x$. Consequently, using Theorem 3 , we obtain the result.

An immediate consequence of Corollary 1 is the generalized Liusternik theorem.

Theorem 4 (Generalized Liusternik theorem). If $h$ is $\left(\|\cdot\|_{1},\|\cdot\|_{2}\right)$ strictly differentiable at the point $x$ in $S$ and $\nabla h(x)$ is surjective then the set $h^{-1}(h(x))$ is tangentially regular at $x$ with respect to the norm $\|\cdot\|_{1}$ and $K_{h^{-1}(h(x))}^{1}(x)=N(\nabla h(x))$.

Proof. Since $h$ is $\|\cdot\|_{2}$ strictly differentiable at $x$ then by the classical Liusternik theorem, we deduce that $K_{h^{-1}(h(x))}^{1}(x)=K_{h^{-1}(h(x))}^{2}(x)=N(\nabla h(x))$.

Let us now prove that $h^{-1}(h(x))$ is tangentially regular at $x$ with respect to the norm $\|\cdot\|_{1}$. Assume without loss of generality that $x=0$ and $h(0)=0$. Its clear that $K_{h^{-1}(0)}^{1}(0) \subset N(\nabla h(x))$. So it suffices to prove $N(\nabla h(0)) \subset T_{h^{-1}(0)}^{1}(0)$.

Fix any element $p$ of $N(\nabla h(0))$ and consider a sequence $x_{r} \rightarrow 0$ in $h^{-1}(0)$ with respect to the norm $\|\cdot\|_{1}$ and $t_{r} \downarrow 0, t_{r}>0$. The previous result shows $h$ is $\|\cdot\|_{1}$ weakly metrically regular at zero, so there is a constant $k$ such that the inequality

$$
d_{h^{-1}(0)}^{1}\left(x_{r}+t_{r} p\right) \leq K\left\|h\left(x_{r}+t_{r} p\right)\right\|
$$

holds for all large $r$, and hence there are points $z_{r} \in h^{-1}(0)$ satisfying the estimate

$$
\left\|x_{r}+t_{r} p-z_{r}\right\|_{1} \leq K\left\|h\left(x_{r}+t_{r} p\right)\right\| .
$$

If we define directions $p_{r}=t_{r}^{-1}\left(z_{r}-x_{r}\right)$ then clearly the points $x_{r}+t_{r} p_{r}$ lie in $h^{-1}(0)$ for large $r$, and since

$$
\left\|p_{r}-p\right\|_{1}=\frac{\left\|x_{r}+t_{r} p-z_{r}\right\|_{1}}{t_{r}} \leq K \frac{\left\|h\left(x_{r}+t_{r} p\right)-h\left(x_{r}\right)\right\|}{t_{r}} \rightarrow k\|\nabla h(0) p\|=0,
$$

we obtain $p \in T_{h^{-1}(0)}^{1}(0)$.

Let us give now an example of a non-strictly Fréchet differentiable function for which the test given in Theorem 3 works.

Example 1. Let $\Omega$ be a bounded domain in $\mathbb{R}^{2}, E=W_{0}^{1,2}(\Omega)$ the Sobolev space with the usual norm $\|\cdot\|_{2}=\|\cdot\|_{W_{0}^{1,2}(\Omega)}$. Let also $p$ and $\varepsilon$ such that $0<\varepsilon<1, \varepsilon+2<$ $p<\infty$. Set $\|\cdot\|_{1}=\|\cdot\|_{L^{p}(\Omega)}$. Note that $\left(E,\|\cdot\|_{2}\right)$ is a separable Banach space. Since $W_{0}^{1,2}(\Omega) \hookrightarrow L^{p}(\Omega)$, it follows that $\left(E,\|\cdot\|_{1},\|\cdot\|_{2}\right)$ is a binormed space such that for some $c>0$, the inequality $\|\cdot\|_{1} \leq c\|\cdot\|_{2}$ holds.

Set $g(u)=|u|^{\varepsilon+2}$ and consider the functional $G$ defined on $E$ by the formula

$$
G(x)=\int_{\Omega} g(x(s)) d s .
$$


Then $G$ is $\|\cdot\|_{2}$ twice Fréchet differentiable at every $x \in E$ and

$$
\begin{aligned}
G^{(1)}(x) h & =\int_{\Omega} g^{\prime}(x(s)) h(s) d s, \\
G^{(2)}(x)\left(h_{1}, h_{2}\right) & =\int_{\Omega} g^{\prime \prime}(x(s)) h_{1}(s) h_{2}(s) d s .
\end{aligned}
$$

Let us note that $G$ is not $\|\cdot\|_{1}$ Fréchet differentiable at any point $x \in E$. Indeed, let $\alpha_{m} \rightarrow \infty$ and $d_{m} \rightarrow \infty$ such that $\left|g\left(d_{m}\right)\right| \geq \alpha_{m}\left|d_{m}\right|^{p}$. By the countable additivity of the Lebesgue measure there exist $C>0$ and $\Omega^{\prime} \subset \Omega$ such that $\mu\left(\Omega^{\prime}\right)>0$, $\operatorname{dist}\left(\Omega^{\prime}, \partial \Omega\right)>0$, and $|x(s)| \leq C$ for all $s \in \Omega^{\prime}$. In this case, put $D=\max \{g(u)$ : $|u| \leq C\}<\infty$. Choose $\Omega_{m} \subset \Omega^{\prime}$ such that $\mu\left(\Omega_{m}\right)=\left|d_{m}\right|^{-p}\left|\alpha_{m}\right|^{-\frac{1}{2}}$ for large $m$.

Let $h_{m}$ be defined by the formula

$$
h_{m}(s)= \begin{cases}d_{m}-x(s) & \text { for } s \in \Omega_{m}, \\ 0 & \text { for } s \in \Omega \backslash \Omega_{m} .\end{cases}
$$

It follows then that $\left\|h_{m}\right\|_{1} \rightarrow 0$ and

$$
\begin{aligned}
\left|G\left(x+h_{m}\right)-G(x)\right| & \geq \alpha_{m}\left|d_{m}\right|^{p} \mu\left(\Omega_{m}\right)-D \mu\left(\Omega_{m}\right) \\
& =\left|\alpha_{m}\right|^{\frac{1}{2}}-D \mu\left(\Omega_{m}\right) \rightarrow+\infty .
\end{aligned}
$$

Let $k_{m} \in C_{0}^{\infty}(\Omega)$ such that $\left\|k_{m}-h_{m}\right\|_{1} \rightarrow 0$. Then $\left\|k_{m}\right\|_{1} \rightarrow 0$, but $G\left(x+k_{m}\right)-$ $G(x) \rightarrow \infty$ since the Lebesgue integral is absolutely continuous. Therefore, $G$ is not $\|\cdot\|_{1}$ Fréchet differentiable at $x$ and consequently, $G$ is not $\|\cdot\|_{1}$ strictly Fréchet differentiable at $x$.

So to test the weak metric regularity of the functional $G$ on a closed set $S$ of $\left(E,\|\cdot\|_{1}\right)$, the classical Theorem 1 cannot be used, but we can apply the Theorem 3 since $G$ is $\|\cdot\|_{2}$ strictly differentiable at $x$. Hence, if $S$ is a closed set in $\left(E,\|\cdot\|_{1}\right)$ such that $\nabla G(x)\left(T_{S}^{1,2}(x)\right)=\mathbb{R}$ then $G$ is $\|\cdot\|_{1}$ weakly metrically regular on $S$ at $x$.

\section{REFERENCES}

[1] M. F. Sukhinin, "Lower semi-Taylor mappings and sufficient conditions for an extremum," Mat. Sb., vol. 182, no. 6, pp. 877-891, 1991.

\section{Author's address}

\section{S. Lahrech}

Department of Mathematics, Faculty of Science, Mohamed First University, Oujda, Morocco

E-mail address: lahrechesciences. univ-oujda.ac.ma 